\title{
HAZARDS OF MULTIPLE MAGNET INGESTION IN CHILDREN;
}

\section{A CASE REPORT}

Dr. Muhammad Ali Sheikh, Dr. Tariq Latif, Dr. Masoom Ali Shah, Dr. Jamil Akhtar, Dr. Abdul Qayuum.

ABSTRACT.....Foreign body ingestion is relatively common in the paediatric population and most object pass through the gastrointestinal tract spontaneously. With the popularity of small magnetic toys, there have been numerous reports of magnet ingestion with morbidity and even mortality. We report a case of 3-years old boy who presented with clinical features of subacute intestinal obstruction with no history of foreign body ingestion. On exploratory laparotomy, he was found to have multiple small bowel perforations due to two small magnets. Magnets were removed and perforations repaired. The aim of this report is to awarepaediatricians of the importance of early surgical referral in case of magnet ingestion, to prevent severe complications.

Key words: Magnet ingestion, children, intestinal perforation.

Article Citation

Sheikh MA, Latif T, Shah MA, Akhtar J, Qayuum A. Hazards of multiple magnet ingestion in children; a case report. Professional Med J 2013;20(4): 634-637.

\section{INTRODUCTION}

Foreign body ingestion, whether inadvertent or intentional is quite common in children.In $80 \%$ of cases children between ages of 6months and 3 years are invoved ${ }^{1-3}$. Commonly ingested objects include coins, toy parts, jewelry, batteries, needles, pins, fish and chicken bones. In majority, these foreign bodies pass through gastrointestinal tract spontaneously ${ }^{4}$. Surgical intervention is required in those cases where foreign object is harmful, like mercurybatteries or if it lodges in certain part of gastrointestinal tract ${ }^{3,4}$. The common sites are lower esophageal sphincter, pylorus and ileocecal valve 4 .

Although most of the ingested foreign bodies pass through the alimentary tract spontaneously, however ingested magnets pose unique challenge ${ }^{4}$. Toysare commonly manufactured for children with powerful earth magnets. These magnets are usually detachable and their small size increases the risk of ingestion by children, who commonly put objects in their mouth. Cases of magnet ingestion with significant morbidity and mortality have been reported in the literature ${ }^{1-5}$. Magnet ingestion has resulted in obstruction, fistula formation, ulceration, perforation and volvulus of intestine ${ }^{2}$. In our part of the world magnetic bracelets and necklaces are worn for their purported healing powers.

The objective of this case report is to aware pediatricians and general public to the dangers associated with multiple magnet ingestion, followed by prompt management to avoid serious sequelae.

\section{CASE REPORT}

A 3- year old boy was brought to the emergency room with lower abdominal pain, vomiting and constipation for last 2 days. Pain was colicky and he had 2-3 episodes of yellow color vomiting. He remained fine in

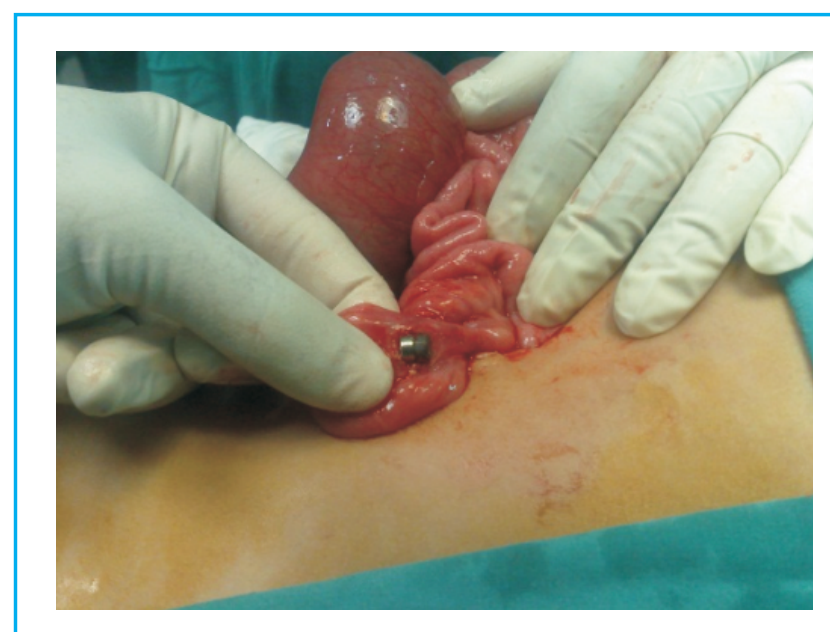

Figure 1. Showing two small magnets joined together causing intestinal perforation. 
between colicky episodes. He had not passed stool for last 5 days. There was no history of foreign body ingestion.

On examination, his pulse was 140 beats/min and temperature was normal. Abdomen was soft and there was mild tenderness in umbilical area. Bowel Sounds were audible and digital rectal examination was unremarkable. Patient was kept nil per oral, fluid resuscitation started and nasogastric tube passed for gastric decompression. His white blood cell count was 17850 with neutrophils of $93.7 \%$ and serum electrolytes were within normal limits. X-ray abdomen showed distended small bowel loops and presence of foreign body was missed because shadow of foreign body was superimposed on shadow of vertebral body. Ultrasound showed streak of fluid in the pelvis. Initially, he improved with conservative management. Doppler ultrasound was performed to rule out malrotation, which showed normal pattern of flow and normal anatomical configuration of superior mesenteric artery and vein .

During 72 hours of conservative management, patient had waxing and waning symptoms of sub acute intestinal obstruction and later deteriorated with visible peristalsis and abdominal guard. Exploratory laparotomy was performed. Per operatively small bowel loops were dilated and matted together by two small magnets. These magnetswere causing obstruction and two sealed perforations in the small bowel. There were three perforations in the mesentery of small bowel as well. Magnets were removed; margins of bowel perforation were freshened and primary repair done. Perforations in the mesentery were also repaired. Post op recovery was uneventful. On 3rd post op day patient was started orally, and discharged on 5th day. On follow up after 6 months he had no complaints.

\section{DISCUSSION}

The incidence of foreign body ingestion is around

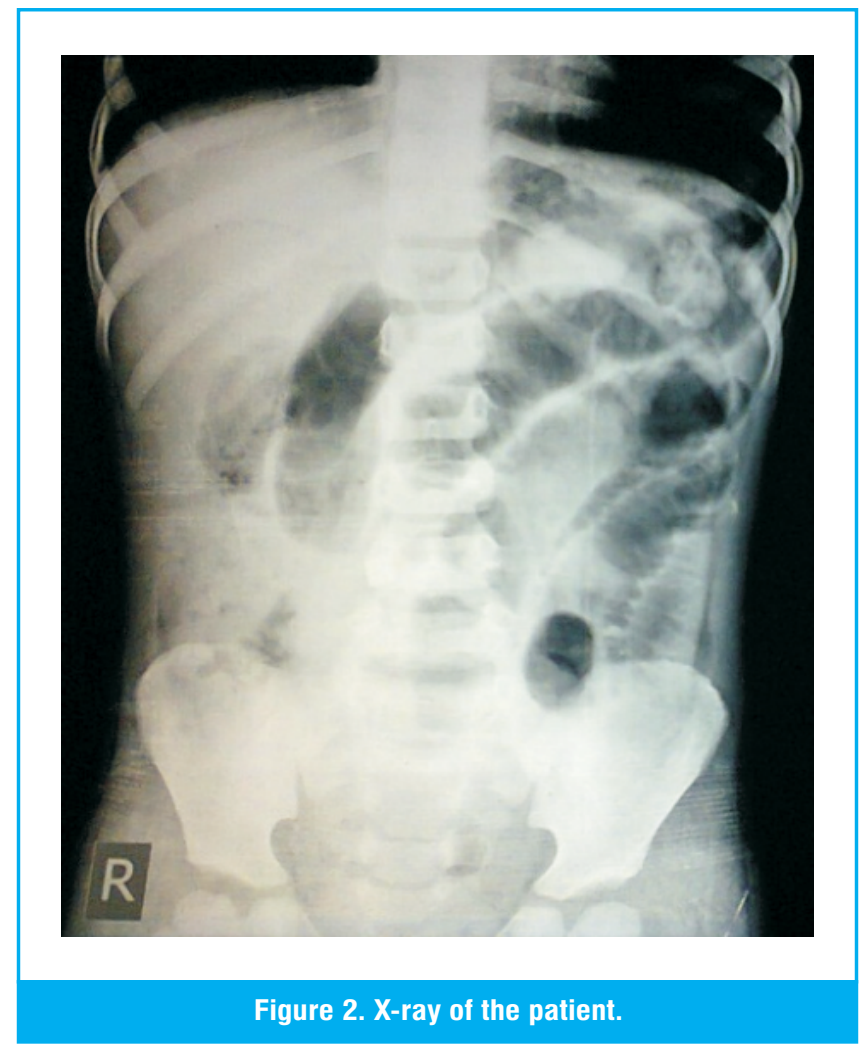

100,000 patients annually in the United States ${ }^{6}$. More than $80 \%$ of the patients are children. Endoscopic retrieval is required in $10-20 \%$ of ingested foreign bodies because of their failure to pass through the esophagus, pylorus or duodenum 6 . Less than $1 \%$ of ingested objects will require surgical intervention ${ }^{3}$. Therefore recommendations for those objects that reach the stomach, are observation and serial abdominal radiograph. The specific scenario of magnet ingestion has not been addressed thoroughly in the literature, because of its low incidence.

A single small magnet will most likely to pass through the gastrointestinal tract without complications. On the other hand, multiple magnets or combination of magnets and metallic objects can become dangerous. Because they can attract each other while residing in different bowel loops, causing compression of the intervening bowel wall. This compression will ultimately lead to pressure necrosis, resulting in perforation, fistulae, and even volvulus, with 
significant morbidity and rarely mortality ${ }^{5}$. A report published in 2006 by Centers for Disease Control and Prevention, identified 1 death and 19 cases of injury to bowel requiring surgical intervention, following magnet ingestion ${ }^{5}$. Bowel perforation was noted in 15 cases. Four patients had bowel obstruction and peritonitis. One fatality caused by volvulus, bowel necrosis, and sepsis was identified.

In our patient there was no history of foreign body ingestion, and initially he improved on conservative management. Secondly shadow of joined magnets was missed on plain X-ray film, because it was superimposed on shadow of lumbar vertebral body. These factors led to delay in surgical intervention in our patient.

Butterworth $\mathrm{J}$ et al reported a case in which a 3 year old boy was discharged from emergency department, as his symptoms settled, although he had clear history of magnet ingestion ${ }^{6}$. Physicians at their institute thought that ingested foreign body was a solitary magnet. But later on it turned out to be case of multiple magnet ingestion and he developed 2 jejunal perforations. Plain radiography lacks the sensitivity to determine the multiplicity of magnetic objects. Therefore, if there is suspicion of magnet ingestion, it should be managed as multiple magnet ingestion until proven otherwise. An attempt should be made for endoscopic retrieval. For magnets out of reach of endoscope, inpatient observation should be undertaken, with serial physical examination and abdominal radiographs. The progression of the magnet down the fecal stream must be ensured until it has passed out of the anal canal. Surgical intervention should be initiated at the first sign of obstruction or increasing abdominal pain. This close observation and early intervention would prevent the more severe complications.

A case presented by Nagaraj and Sunil described how a magnet ingested with metallic objects could also become dangerous ${ }^{7}$. They described a 10 year old boy ingested an alkaline disk battery, a magnet and a steel ball. On examination, he was found to have signs of peritonitis. On exploration, magnet and disk battery were attached across a loop of ileum, causing necrosis and perforation. The patients with even single magnet ingestion should be counseled not to wear clothing with metallic buttons or buckles that overlay

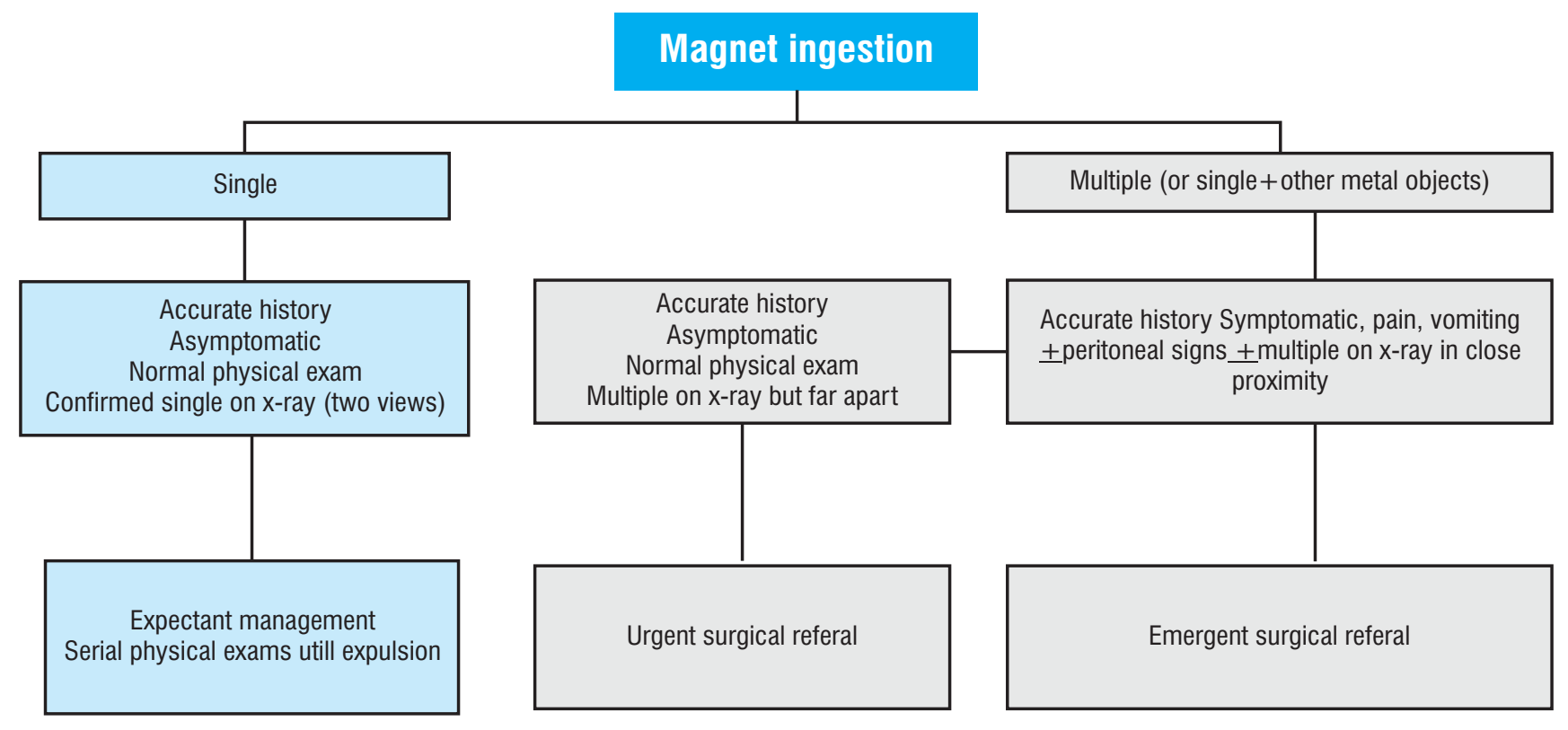


the abdomen for prolonged periods, till the magnet is passed out ${ }^{8}$.

Pressure necrosis and fistula formation could be a gradual process. In older children, omental walling can limit peritoneal inflammation resulting in minimal physical examination findings. The absence of significant symptoms and signs of intestinal damage should not preclude aggressive management. Ahmed A et al reported a 5 year old girl who had multiple admissions in the hospital for pain abdomen and discharged home, assuming the foreign body would pass spontaneously 9 . After 2 months she developed intestinal obstruction, and on exploration, was found to have cecojejunal fistula. Childrenless than 3 years have relatively fat devoid omentum, which may be less effective in containing perforation, so these patients tend to present early with signs of peritonitis.

In conclusion magnet ingestion should be managed aggressively. An algorithm has been proposed for their management. Table-I. If a solitary magnet is ingested then at least two views of plain $\mathrm{x}$-rays should be obtained. In case of multiple magnet ingestion patient should be hospitalized and have serial examinations. All paediatricians and healthcare providers involved in management of children should be aware of the problems caused by multiple magnet ingestion.

Copyright@ 26 Apr, 2013

\section{REFERENCES}

1. Dutta $S$, Barzin A. Multiple magnet ingestion as a source of severe gastrointestinal complications requiring surgical intervention. Arch Pediatric Adolesc Med 2008; 162(2): 123-125.

2. Devenyi AG. Hazards of ingested magnets. Jr Lancaster Gr Hos 2009; 4(3):90-93.

3. Wong HL, Phillips BA. Opposites attract: a case of magnet ingestion. CJEM 2009; 11(5):493-495.

4. Cauchi JA, Shawis RN. Multiple magnet ingestion and gastrointestinal morbidity. Arch Dis Child 2002; 87:539-540.

5. Centers for Disease Control and Prevention (CDC). Gastrointestinal injuries from magnet ingestion in children: United States, 2003-2006. MMWR Morb Mortal Wkly Rep. 2006; 55(48):1296-1300.

6. Butterworth $\mathrm{J}$, Feltis B. Toy magnet ingestion in children: Revising the algorithm. J PediatrSurg 2007; 42:E3-E5.

7. Nagaraj HS, Sunil I. Multiple foreign body ingestion and ileal perforation. Pediatr Surg Int. 2005; 21(9):718-720.

8. Salimi A, Kooraki S, Esfahani SA, Mehdizadeh M. Multiple magnet ingestion: Is there a role for early surgical intervention? Ann Saudi Med. 2012 Jan; 32(1):93-6.

9. Ahmed AM, Hassab MH, Al-Hussaini AA, Al-Tokhais TI. Magnetic toy ingestion leading to jejunocecal fistula in a child. Saudi Med J. 2010 Apr; 31(4):442-4.

\section{Dr. Abdul Qayuum}

Department of Paediatric Surgery,

Shaikh Zayed Postgraduate Medical Institute, Lahore.

Correspondence Address:

Dr. Muhammad Ali Sheikh

Department of Paediatric Surgery,

Shaikh Zayed Postgraduate Medical Institute, Lahore. drali444@yahoo.com

3. DR. MASOOM ALI SHAH

Department of Paediatric Surgery,

Shaikh Zayed Postgraduate Medical Institute, Lahore.

4. Dr. Jamil Akhtar

Department of Paediatric Surgery,

Shaikh Zayed Postgraduate Medical Institute, Lahore. 